\title{
On sait enfin traiter un infarctus cérébral !
}

\author{
We Finally Know how to Cure Cerebral Infarction!
}

\author{
M. Freysz \\ (C) SFMU et Lavoisier SAS 2015
}

Trois articles de ce numéro des Annales françaises de médecine d'urgence sont consacrés à l'accident vasculaire cérébral (AVC) ischémique. L'un, une revue générale sur la thrombectomie et ses conséquences sur la filière de soins [1], et deux autres, l'un portant sur une filière de soins dans un CHU [2] et le dernier sur une évaluation des délais d'admission aux urgences d'hôpitaux généraux [3]. Le mot d'ordre est de gagner du temps pour reperfuser le cerveau. En effet, chaque minute qui passe sans reperfusion aggrave considérablement les séquelles, « le temps, c'est du cerveau ».

Plus de dix ans après les premières preuves sur l'efficacité de la thrombolyse, l'année 2015 a permis de prouver l'intérêt de la thrombectomie dans l'infarctus cérébral, ce qui devrait désormais diminuer considérablement les séquelles sévères encore trop fréquentes. L'accident vasculaire cérébral reste la première cause de handicap moteur, la deuxième cause de handicap cognitif et la troisième cause de mortalité. L'utilisation de la thrombolyse intraveineuse précoce a déjà permis des avancées majeures avec, en particulier, une meilleure maîtrise des contre-indications liées à l'âge, ainsi qu'une modification de l'autorisation de mise sur le marché (AMM) européenne pour permettre de développer la téléfibrinolyse par l'urgentiste. Mais la thrombolyse est très souvent inefficace dans les ischémies artérielles du tronc basilaire ou du segment M1 de la sylvienne, ce qui génère mortalité et séquelles les plus lourdes. Ceux d'entre nous qui avaient vu les résultats cliniques après thrombectomie étaient déjà presque convaincus ; mais c'est au début de cette année que l'étude MR CLEAN a confirmé de manière indiscutable son intérêt, corroboré depuis par de nombreuses autres études randomisées. Notons que MR CLEAN est une étude clinique néerlandaise, rendue possible et optimisée par le financement de la thrombectomie

M. Freysz ( $\square)$

Département de Médecine d'Urgence,

Faculté des sciences de la santé Médecine,

Université de Bourgogne,

21079 Dijon cedex

e-mail : marc.freysz@chu-dijon.fr aux hôpitaux avec obligation de participer à l'étude. Les contraintes donnent parfois des résultats extraordinaires pour la recherche clinique. La thrombectomie nécessite un plateau technique disponible $24 / 7$ avec surtout un neuroradiologue entraîné, ce qui va bouleverser l'organisation actuelle des filières de soins [1]. Cette nouvelle technique nécessite en effet une adaptation région par région, pour que l'ensemble des patients bénéficient de ce progrès thérapeutique majeur. Nous sommes, en régulation médicale, l'élément moteur du gain de temps. L'intérêt de la préalerte AVC au service receveur (unité neurovasculaire [UNV] ou accueil des urgences) est déjà démontré. Il faudra maintenant affiner l'interrogatoire du patient et/ou de la famille pour choisir le juste lieu d'adressage hospitalier. En fonction de la gravité de la symptomatologie initiale, le score NIHSS est bien entendu d'une aide précieuse, mais il semble évident qu'en fonction de la région et du temps de trajet (avec pour le transport héliporté, le rôle majeur de la météorologie), le rôle pivot de la régulation médicale se trouve encore renforcé. Voilà un sujet où notre organisation pourrait être source de gain de temps pour nos patients. Cela a déjà été démontré pour le syndrome coronarien aigu et la traumatologie grave, autres pathologies tempsdépendantes. À nous tous d'être moteur de ce changement et de montrer son intérêt.

Le deuxième article est consacré à une filière de soins très spécifique, dans un $\mathrm{CHU}$ multisite dans lequel accueil des urgences et neurologie ne sont pas (encore ?) réunis : l'organisation de la filière neurovasculaire est dépendante du plateau technique et des structures hospitalières locaux [2].

Le délai médian d'arrivée à l'hôpital, 5h15 dans l'article consacré à l'arrivée des patients en hôpital général sans structure UNV [3], s'explique pour partie par le retard à l'appel au 15. Deux éléments y contribuent : la difficulté de sensibiliser la population aux signes précoces de l'AVC résumés dans l'acronyme «face, arm, speech, time » (FAST) mais également les campagnes d'information du grand public encore insuffisamment perçues, lesquelles doivent être répétées régulièrement et encore plus fréquemment par notre ministère de la Santé. 


\section{Références}

1. Gory B, Lehot JJ, Gueugniaud PY, et al (2015) Thrombectomie mécanique de l'infarctus cérébral : pourquoi une prise en charge ultrarapide est nécessaire ? Ann Fr Med Urg 5:252-9

2. Vannier-Bernard S, Debiais S, Pinçon O, et al (2015) Filière neurovasculaire : évaluation des facteurs de non-admission directe des accidents vasculaires cérébraux (AVC) en unité neurovasculaire (UNV) et conséquences de l'orientation initiale sur l'accès à la thrombolyse intraveineuse. Ann Fr Med Urg 5:207-13

3. Redjaline A, Perrillat Y, Marrone G, et al (2015) Délais d'admission et profil des patients présentant un accident vasculaire cérébral admis aux urgences d'hôpitaux ne disposant pas d'une unité neurovasculaire. Ann Fr Med Urgence 5:214-22 